\title{
MicroRNA-126 modulates angiogenesis and tube formation through enhancing epidermal growth factor- like domain 7 expression and phosphorylating PI3K/AKT signaling pathway
}

\author{
Type \\ Research paper \\ Keywords \\ angiogenesis, PI3K/AKt signaling pathway, microRNA-126, epidermal growth factor-like domain 7 , \\ formation of tube
}

\begin{abstract}
Introduction

Dysregulated angiogenesis is a critical characteristic for endothelial dysfunction disorders. This study aimed to determine functions of microRNA-126 in formation of tube and investigated the potential mechanisms.
\end{abstract}

\section{Material and methods}

The synthesized microRNA-126 control and microRNA-126 inhibitor plasmids were transfected into human umbilical-vein endothelial cells (HUVECs) using lipofectamine 2000 reagent. Cell counting kit-8 (CCK-8) was employed to measure proliferative capability of HUVECs. Transwell analysis was used to evaluate HUVECs invasive ability. Real time PCR (RT-PCR) was utilized to access epidermal growth factor-like domain 7 (EGFL7) and microRNA-126 mRNA transcription. Tubeforming capability in HUVECs was determined. Dual-luciferase assay and linear-regression analysis were conducted to measure interaction between EGFL7 and microRNA-126 molecule.

Phosphoinositide-3-kinase/protein kinase-B (PI3K/AKT) signaling pathway associated molecules were evaluated using western blot assay.

\section{Results}

Silencing of microRNA-126 significantly enhanced proliferative capability and invasive ability of HUVECs compared to those of microRNA-126 control group $(p<0.05)$. microRNA-126 silencing remarkably promoted tube formation and significantly up-regulated EGFL7 compared to those of microRNA-126 control group ( $p<0.05$ ). microRNA-126 could interact with EGFL7 molecule. microRNA-126 was also negatively correlated with EGFL7 molecule in HUVECs $(p<0.05)$. Silencing of microRNA-126 significantly enhanced p-PI3K/PI3K ratio compared to that of microRNA-126 control group $(p<0.05)$. microRNA-126 silencing also remarkably increased $p-A K T / A K T$ ratio compared to that of microRNA-126 control group $(p<0.05)$.

\section{Conclusions}

microRNA-126 modulated angiogenesis and tube formation through increasing EGFL7 expression and phosphorylating PI3K/AKT signaling pathway. 
MicroRNA-126 modulates angiogenesis and tube formation through enhancing epidermal growth factor-like domain 7 expression and phosphorylating PI3K/AKT signaling pathway

Running title: Silencing of microRNA-126 promotes tube formation 


\section{Abstract}

Introduction: Dysregulated angiogenesis is a critical characteristic for endothelial dysfunction disorders. This study aimed to determine functions of microRNA-126 in formation of tube and investigated the potential mechanisms. Materials and methods: The synthesized microRNA-126 control and microRNA-126 inhibitor plasmids were transfected into human umbilical-vein endothelial cells (HUVECs) using lipofectamine 2000 reagent. Cell counting kit-8 (CCK-8) was employed to measure proliferative capability of HUVECs. Transwell analysis was used to evaluate HUVECs invasive ability. Real time PCR (RT-PCR) was utilized to access epidermal growth factor-like domain 7 (EGFL7) and microRNA-126 mRNA transcription. Tube-forming capability in HUVECs was determined. Dual-luciferase assay and linear-regression analysis were conducted to measure interaction between EGFL7 and microRNA-126 molecule. Phosphoinositide-3-kinase/protein kinase-B (PI3K/AKT) signaling pathway associated molecules were evaluated using western blot assay.

Results: Silencing of microRNA-126 significantly enhanced proliferative capability and invasive ability of HUVECs compared to those of microRNA-126 control group $(p<0.05)$. microRNA-126 silencing remarkably promoted tube formation and significantly up-regulated EGFL7 compared to those of microRNA-126 control group $(p<0.05)$. microRNA-126 could interact with EGFL7 molecule. microRNA-126 was also negatively correlated with EGFL7 molecule in HUVECs $(p<0.05)$. Silencing of microRNA-126 significantly enhanced $\mathrm{p}-\mathrm{PI} 3 \mathrm{~K} / \mathrm{PI} \mathrm{KK}$ ratio compared to that of microRNA-126 control group $(p<0.05)$. microRNA-126 silencing also remarkably increased $p-A K T / A K T$ ratio 
compared to that of microRNA-126 control group $(p<0.05)$.

Conclusions: microRNA-126 modulated angiogenesis and tube formation through increasing EGFL7 expression and phosphorylating PI3K/AKT signaling pathway.

Keywords: angiogenesis; microRNA-126; epidermal growth factor-like domain 7; PI3K/AKT signaling pathway; tube formation 


\section{Introduction}

Clinically, the dysregulated angiogenesis is a critical characteristic for the endothelial dysfunction of many disorders [1-3], such as ischemic myocardium and stroke. The structural integrity and functional completeness of blood vessel are important for maintaining health of the vascular system [4]. Moreover, there are also many other disorders involving damages of endothelial cells (ECs) and dysregulated angiogenesis, such as aging-associated macular degeneration, malignant tumors and degenerative disorders $[5,6]$. Therefore, when individuals suffering from the above diseases, the ECs would mobilize the generation of novel vessels, through invading extra-cellular matrix and proliferating into lumen [7].

In physiological state, ECs usually maintain the relatively lower proliferative capability. However, when ESs suffering from pathological stimuli, proliferation is enhanced and neovascularization is initiated by up-regulating production of growth factors and cytokines $[8,9]$. Due to the crucial functions of ECs in integrity of blood vessel and tube formation [10,11], ECs are extensively studied for discovering the novel therapeutic strategies. Human umbilical-vein endothelial cells (HUVECs), as the most common ECs, is characterized by high capability of tube formation and angiogenesis $[12,13]$. Thus, it's a good approach to discover the therapeutic or targeting molecules for treating stroke or ischemic reperfusion, by exploring their regulative effects on ECs.

MicroRNAs (miRNAs), as non-coding and single strand RNAs, are consistent of a series of 18 to 24 nucleotides and could inhibit post-transcriptional processes and modulate gene expression [14,15]. miRNAs could also regulate cell growth, differentiation, cell apoptosis, organ 
development and metabolic procedures [16]. Furthermore, miRNAs could modulate angiogenesis by regulating tube formation [17], promote angiogenic responses [18] and improve vascular development in endothelial cells [19]. Among multiple miRNAs, microRNA-126 (miR-126) has been proven to be correlated with the angiogenesis and vascular integrity [20,21]. Epidermal growth factor like domain 7 (EGFL7) is considered as potential target of miR-126 and expresses during the pathological processes of blood vessels $[22,23]$. However, whether miR-126 affecting the tube formation of HUVECs through modulating EGFL7 molecules or the other pathways has never to be clarified till now. Therefore, the present study was aimed to investigate effects of miR-126 silencing on tube formation in HUVECs.

\section{Materials and methods}

\section{HUVECs culture}

HUVECs (American Type Culture Collection, Manassas, Virginia, USA) were cultured in Dulbecco's modified eagle's medium (DMEM, Gibco BRL. Co. Ltd., Grand Island, New York, USA) containing 10\% fetal bovine serum (FBS, Gibco BRL. Co. Ltd.) and supplementing with $1 \%$ penicillin-streptomycin (Beyotime Biotech., Shanghai, China) in $5 \% \mathrm{CO}_{2}$ at $37^{\circ} \mathrm{C}$. The present study has been approved by Ethics Committee of The Second Affiliated Hospital of Hainan Medical University, Haikou, China.

\section{microRNA-126 inhibitor synthesis and transfection into HUVECs}

The microRNA-126 inhibitor was synthesized using the following oligonucleotides: 5'-CGCAUUAUUACUCACGGUACGA-3'. Meanwhile, the 
sham control for microRNA-126 oligonucleotides (microRNA-126 control) was also synthesized as the following oligonucleotides: 5'-CAGUACUUUUGUGUAG UACAA-3'. The HUVECs were seeded and cultured to the density of $1 \times 10^{5}$ cells $/ \mathrm{ml}$. Then, the above microRNA-126 inhibitor or microRNA-126 control was transfected into HUVECs using Lipofectamine 2000 (Cat. No. 11668-027, Invitrogen/Life Technologies, Carlsbad, CA, USA) as instructed by the protocol of manufacturer.

\section{Cell counting kit-8 (CCK-8) assay}

Cell proliferative viability of HUVECs was examined using CCK-8 commercial kit (Cat. No. C0038, Beyotime Biotech.) based on protocol of the manufacturer. Briefly, HUVECs were cultured to the dosage of $1 \times 10^{5} \mathrm{cells} / \mathrm{ml}$ and treasfected with microRNA-126 inhibitor and microRNA-126 control plasmids. Then, the transfected HUVECs were cultured for $24 \mathrm{~h}$ and administrated with CCK-8 regent for $4 \mathrm{~h}$ at $37{ }^{\circ} \mathrm{C}$ (at final dosage of $10 \mu \mathrm{l} / \mathrm{ml}$ ). At last, proliferative viabilities of HUVECs were evaluated using a micro-plate reader (Mode: MCC/340, Thermo Fisher Scientific, Hudson, NH, USA) at 450 $\mathrm{nm}$.

\section{Transwell assay}

HUVECs were seeded and cultured to the density of $1 \times 10^{5}$ cells $/ \mathrm{ml}$, and then transfected with microRNA-126 inhibitor or microRNA-126 control plasmid. Then, HUVECs were added to lower chamber of transwell system (Corning-Costar, Corning, NY, USA) and cultured with DMEM supplementing with $10 \%$ FBS. Cell supernatants of HUVECs were allowed to migrate to the 
upper chamber and mixed with HUVECs (un-transfected) in upper chamber for $48 \mathrm{~h}$ at $37{ }^{\circ} \mathrm{C}$. Subsequently, HUVECs in upper chamber were fixed using $4 \%$ paraformaldehyde (Beyotime Biotech.) for $20 \mathrm{~min}$, washed with phosphate buffered saline (PBS, Beyotime Biotech.) and stained with 1\% crystal violet (weight/volume) (Sigma-Aldrich) for 5 min. Finally, amounts of HUVECs were counted with an ECLIPSE-Ti inverted microscope (Nikon, Tokyo, Japan).

\section{Tube forming assay}

Tube formation was evaluated as the described protocol of the former study [24] with some minor modifications. In brief, total of $200 \mu$ l Matrigel (BD Biosciences, San Jose, CA, USA) was added into a well of 24-well plate (Corning-Costar), with gently shaking for whole culture periods. Subsequently, HUVECs suspension was added into wells at concentration of $1 \times 10^{6}$ cells/well. Then, HUVECs were treated using the supernatant isolated from microRNA-126 inhibitor and microRNA-126 control plasmids transfected HUVECs, and cultured at $37{ }^{\circ} \mathrm{C}$ for $24 \mathrm{~h}$. Eventually, the formed tubes were evaluated and counted with an ECLIPSE-Ti inverted microscope (Nikon, Tokyo, Japan). The formed tube amounts were used to reflect the tube-forming capability in HUVECs.

\section{$R T-P C R(R T-P C R)$ assay}

Total RNAs of HUVECs were extracted using Trizol regents (Beyotime Biotech.), while the complementary DNAs (cDNAs) were synthesized and generated using commercial Reverse Transcription (RT) Kit (Western Biotech. Co. Ltd., Chongqing, China) as described by the protocol of manufacturer. The 
mRNA transcriptions of EGFL7 and microRNA-126 gene were obtained with a Sybr-Green I PCR regent (Western Biotech. Co. Ltd.), using specific primers illustrated in Table I. Relative mRNA transcriptions of EGFL7 and microRNA-126 gene were evaluated with a GDS8000 professional Gel-Scanning System (UVP, Sacramento, CA, USA) and analyzed as the previously published $2^{-\triangle \Delta \mathrm{Ct}}$ approach described [25].

\section{Dual luciferase assay}

Interaction between EGFL7 mRNA and miR-126 mRNA was analyzed using Dual Luciferase assay in commercial 293T cells (Shanghai Cell Bank of CAS, Shanghai, China). The applied microRNA-126 mimic, microRNA-126 control, pMIRGLO-EGFL7-Mut and pMIRGLO-EGFL7-WT were synthesized as described by our previous report [26]. In this study, luciferase activity was measured with a Dua-Luciferase Reporter Assay System (Cat. No. E1910, Promega, Madison, MI, USA) as instructed by protocol of manufacturer. Luciferase activity was analyzed and calculated through comparing with the activity of Renilla luciferase plasmid (Promega, Madison, MI, USA).

\section{Western blot assay}

HUVECs were lysed with a radioimmunoprecipitation assay solution (RIPA, Beyotime Biotech.) and centrifuged at speed of $10000 \mathrm{r} / \mathrm{min}$ for $10 \mathrm{~min}$ at $4{ }^{\circ} \mathrm{C}$. The concentration of lysed protein was measured using bincinchonic acid (BCA) Protein detection Kit (Cat. No. P0010, Beyotime Biotech.) based on the protocol of manufacturer. Then, protein was separated using $12 \%$ sodium-dodecyl sulfate-polyacrylamide gel electrophoresis (SDS-PAGE) and 
transferred onto the commercial polyvinylidene fluoride membrane (PVDF, Cat. No. FFP24, Beyotime Biotech.) using the 170-3940 Semi-Dry Electrophoretic Transfer Device (Bio-Rad Laboratories). The antibodies, including anti-EGFL7 (Cat. No. ab102796, 1:2000), anti-phosphatidylinositol 3-kinase (PI3K) (Cat. No. ab32089, 1:2000), anti-phosphorylated PI3K (p-PI3K, Cat. No. ab182651, 1:3000) and anti-AKT (Cat. NO. ab185633, 1:2000), anti-phosphorylated AKT (p-AKT, Cat. No. ab81283, 1:2000) and anti- $\beta$-actin (1: 1000; Cat. No. ab228001, 1:3000) were utilized to incubate the PVDF overnight at $4{ }^{\circ} \mathrm{C}$. Subsequently, PVDF membranes were washed using PBS and treated with the horseradish peroxidase (HRP)-labeled secondary antibody (Cat. No. ab6721, 1:1000) for $2 \mathrm{~h}$ at room temperature. All of the above antibodies were purchased from Abcam Biotech. Co. Ltd. (Cambridge, Massachusetts, USA). Finally, the PVDF membranes were treated using BM-chemiluminescence Kit (Cat. No. 11520709001, Sigma-Aldrich.) at room temperature for 2 min in dark. The western blot band was evaluated and analyzed using Labworks ${ }^{\mathrm{TM}}$ Analaysis Software 4.0 (Labworks, Upland, CA, USA).

\section{Statistical analysis}

In this study, data were defined as mean \pm standard deviation and analyzed using professional SPSS software (version: 20.0, SPSS Inc., Chicago, UII, USA). Tukey's post-hoc test validated analysis of variance (ANOVA) was employed to compare the statistical differences among the multiple groups. Liner correlation analysis was used to analyze correlation between EGFL7 mRNA transcriptions and miR-126 mRNA transcriptions. All of the experiments or tests were conducted in triplicate in this study. $A p<0.05$ 
was defined as the statistical difference.

\section{RESULTS}

\section{Silencing of microRNA-126 enhanced proliferative capability of HUVECs}

To verify effects of microRNA-126 silencing on proliferative capability of HUVECs, we performed the CCK-8 assay. Our results indicated that silencing of microRNA-126 (microRNA-126 inhibitor group) remarkably enhanced proliferative capability of HUVECs comparing to that of microRNA-126 control group (Figure 1A, $p<0.05$ ). Meanwhile, there was no significant difference for cell viability between microRNA-126 control group and HUVECs group (Figure $1 \mathrm{~A}, p>0.05)$. Moreover, the results also identified that the proliferative effect of microRNA-126 on HUVECs demonstrated a time-dependent manner, but without significant differences among different time points (Figure 1B).

\section{Silencing of microRNA-126 increased invasive ability of HUVECs}

Due to the enhancive effects of microRNA-126 silencing on cell viability, the effects of which on cell invasion was also examined in this study (Figure 1C). The findings showed that Silencing of microRNA-126 remarkable increased invasive ability of the HUVECs compared to that of microRNA-126 control group (Figure 1D, $p<0.05$ ).

\section{microRNA-126 silencing promoted the tube formation of HUVECs}

The tube forming findings demonstrated that there were plenty of integrated tubes in the microRNA-126 inhibitor group, however, even no obvious integrated tubes in HUVECs and microRNA-126 control group (Figure 
2A). According to the statistical findings, the amounts of integrated tube of the microRNA-126 inhibitor group were significantly more compared to those of microRNA-126 control group (Figure 2B, $p<0.05$ ).

\section{Silencing of microRNA-126 up-regulated EGFL7 expression}

In the microRNA-126 inhibitor transfected HUVECs, the transcription of microRNA-126 mRNA was significantly lower compared to that in microRNA-126 control group (Figure 3A, p<0.05). Interestingly, the EGFL7 mRNA transcription in microRNA-126 inhibitor group was remarkably higher compared to that in the microRNA-126 control group (Figure $3 \mathrm{~B}, p<0.05$ ). Meanwhile, the western blot results also showed that microRNA-126 inhibitor treatment significantly enhanced EGFL7 expression compared to that of microRNA-126 control group (Figure 4A, $p<0.05$ ). Therefore, there might a relationship between microRNA-126 molecule and EGFL7 molecule.

\section{microRNA-126 is correlated with EGFL7 molecule}

The Dual luciferase assay results indicated that co-transfection of pMIRGLO-EGFL7-WT and microRNA-126 control remarkably decreased the Firefly/Renilla luciferase ratio, comparing to the co-transfection of pMIRGLO-EGFL7-WT and microRNA-126 mimic (Figure 4B, $p<0.05$ ). Meanwhile, the linear regression analysis findings showed that microRNA-126 expression was negatively correlated with EGFL7 expression (Figure 4C, $p<0.05)$ 


\section{Silencing of microRNA-126 promoted phosphorylation of PI3K}

In this study, the PI3K and p-PI3K expressions were also evaluated using the western blot analysis (Figure 5A). The results identified that $p$-PI3K/PI3K ratio was remarkably promoted in microRNA-126 inhibitor group compared to that of the microRNA-126 control group (Figure 5B, $p<0.05$ ). However, there was no remarkable difference for the $\mathrm{p}-\mathrm{PI}$ IK/PI3K ratio between HUVECs group and microRNA-126 control group (Figure 5B, $p>0.05$ ).

\section{microRNA-126 silencing enhanced phosphorylation of AKT}

We also determined the expressions of PI3K and p-PI3K using western blot assay (Figure 6A). Our results identified that microRNA-126 inhibitor transfection remarkably enhanced the ratio of $\mathrm{p}-\mathrm{PI} 3 \mathrm{~K} / \mathrm{PI} \mathrm{K}$ compared to that of microRNA-126 control transfected HUVECs (Figure 6B, $p<0.05$ ). However, no obvious difference was discovered for the p-PI3K/PI3K ratio between HUVECs and microRNA-126 control group (Figure 5B, $p>0.05$ ).

\section{Discussion}

Angiogenesis has been proven to participate in plenty of intracellular processes, such as proliferation and migration of endothelial cells, formation of tube, and even regeneration of the matrix $[27,28]$. The former study [29] reported that EGFL7 molecule plays critical roles in many human ECs associated diseases, such as gastrointestinal diseases and hepatocellular disorders. The microRNAs, such as microRNA-377 and microRNA-126 also contribute to a series of endogenous regulations of endothelial cells, such as modulation of angiogenesis and regulation of tube formation $[18,30]$. Our 
current findings demonstrated that the microRNA-126 silencing enhanced proliferative capability and invasive ability and was expressed along with the expression of EGFL7 in HUVECs. These results are consistent with the former investigations $[31,32]$ that investigating the biological effects of microRNA-126 on cells. However, the associated biological mechanism for the microRNA-126 and its targeting modulation have never been clarified in HUVECs.

To further explore effects of microRNA-126 silencing on novel blood vessel generation, the tube forming assay has performed to determine effects of microRNA-126 silencing on tube formation or the angiogenic functions. The findings demonstrated that microRNA-126 silencing remarkably enhanced formation of tube comparing to the control group. However, there are also a few microRNAs, such as microRNA-1224, illustrate different effects on the tube formation [33]. Therefore, the specific mechanisms for the microRNAs triggered tube formation also need to be further explored in the following studies.

Andersen et al. [34,35] reported that the microRNA-126 is associated with the EGFL7 molecule, therefore, we determined correlation between microRNA-126 and EGFL7 molecule, using both dual luciferase assay and linear regression analysis. Firstly, the findings here indicated that microRNA-126 silencing remarkably enhanced EGFL7 expression in HUVECs, which illustrates the equal results with former report [36]. Both of dual luciferase assay and linear regression analysis results showed that microRNA-126 could interact with EGFL7 gene, which hints that microRNA-126 may involve in formation of tube through interacting with and modulating the EGFL7 gene expression. 
Furthermore, according to the previous studies [26,37], PI3K/AKT signaling pathway associated molecules have been proven to involve in the processes of tube formation. Therefore, PI3K/AKT and the phosphorylated $\mathrm{PI} 3 \mathrm{~K} / \mathrm{AKT}$ states were evaluated in the microRNA-126 inhibitor transfected HUVECs. The findings demonstrated that microRNA-126 silencing regulated formation of tube by enhancing the ratio of $p-P I 3 K / P I 3 K$ and $p-A K T / A K T$. Our findings hint that silencing of microRMA-126 promotes the formation of tube via enhancing the phosphorylation of $\mathrm{PI} K \mathrm{~K}$ and AKT levels in PI3K/AKT signaling pathway. Therefore, we speculated that the microRNA-126 might be an anti-angiogenetic microRNA, however, which needs to be proven by more experimental data in following studies. Moreover, microRNA-126 also involves in development and progression of many endothelial-related diseases, such as cancers, diabetes, coronary heart disease [38-40]. However, the specific mechanism for roles of microRNA-126 have also not been fully clarified. Might be this study proved effects of microRNA-126 would be helpful to study the above diseases.

Moreover, through this study, we also identified that analysis of circulating DNA could act as a novel approach for detecting the micorRNAs and the other biomarkers [41-43]. Especially, analysis for the circulating DNA is beginning to have great importance in tumors where it is possible to analyze circulating tumor DNA to identify biomarkers, such as microRNAs. Take the present study for example, the microRNA-126 could modulate the angiogenesis. Therefore, circulating DNA analysis is useful to define the diagnosis and prognosis of diseases.

In conclusion, microRNA-126 silencing enhanced proliferative capability 
and invasive ability, and promote tube formation in HUVECs. Meanwhile, the above effects of microRNA-126 might be associated with interaction of EGFL7 molecule and modulation of phosphorylated PI3K/AKT. In summary, our study proved for the first time that silencing of microRNA-126 enhances formation of tube formation in HUVECs through interacting with EGFL7 molecule and modulating PI3K/AKT signaling pathway. 


\section{Table legends}

Table I . Gene sequences for the RT-PCR assay.

\section{Figure legends}

Figure 1. Effects of microRNA-126 inhibitor administration on proliferative capability and invasive ability of HUVECs. A. Statistical analysis for microRNA-126 inhibitor's effects on proliferative capability. B. Proliferative effect of microRNA-126 on HUVECs with a time-dependent manner. C. Images of the invasive HUVECs. D. Statistical analysis for microRNA-126 induced cell invasion. ${ }^{*} p<0.05$ vs. microRNA-126 control group. Magnification, 100×.

Figure 2. microRNA-126 inhibitor transfection induced the formation of tube. A. Tube formation images in the cultured HUVECs. B. Statistical analysis of tube formation. " $p<0.05$ vs. microRNA-126 control group. Magnification, $100 \times$.

Figure 3. microRNA-126 inhibitor administration enhanced the EGFL7 mRNA expression. A. Statistical analysis of microRNA-126 mRNA expression. B. Statistical analysis of EGFL7 mRNA expression. " $p<0.05$ vs. microRNA-126 control group.

Figure 4. Relationship between microRNA-126 and EGFL7 molecule. A. Determination for effects of microRNA-126 inhibitor administration on EGFL7 expression using western blotting assay. B. Dual-luciferase assay for evaluating interaction between microRNA-126 gene and EGFL7 gene. C. Correlation between microRNA-126 and EGFL7 using Linear Regression analysis. " $p<0.05$ vs. microRNA-126 control group. ${ }^{*} p<0.05$ vs. microRNA-126 mimic+EGFL7-WT group.

Figure 5. Evaluation for enhancive effects of microRNA-126 inhibitor 
administration on $\mathrm{p}-\mathrm{PI} 3 \mathrm{~K}$ and PI3K expression using western blotting assay. A. Western blotting images for $\mathrm{p}-\mathrm{PI} 3 \mathrm{~K}$ and PI3K expression. B. Statistical analysis for ratio of $\mathrm{p}-\mathrm{PI}$ KK/PI3K. " $p<0.05$ vs. microRNA-126 control group.

Figure 6. microRNA-126 silencing promoted p-AKT and AKT expression using western blotting assay. A. Western blotting images for p-AKT and AKT expression. B. Statistical analysis for ratio of $p$-AKT/AKT. " $p<0.05$ vs. microRNA-126 control group. 


\section{References}

1. Aldabbous I, Abdul-Salam V, McKinnon T, et al. Neutrophil extracellular traps promote angiogenesis: evidence from vascular pathology in pulmonary hypertension. Arterioscler Thromb Vasc Biol 2016;36:2078-2087.

2. Ekiz-Bilir B, Bilir B, Aydin M, et al. Evaluation of endocan and endoglin levels in chronic kidney disease due to diabetes mellitus. Arch Med Sci 2019; 15: 86-91.

3. Zhao T, Zhao W, Chen Y, et al. Vascular endothelial growth factor (VEGF)-A: role on cardiac angiogenesis following myocardial infarction. Microvasc Res 2010;80:188-194.

4. Sato Y. Double-face of vasohibin-1 for the maintenance of vascular homeostasis and healthy longevity. J Atheroscler Thromb 2018;25:461-466.

5. Zhou Q, Anderson $\mathrm{C}$, Zhang $\mathrm{H}$, et al. Repression of choroidal neovascularization through actin cytoskeleton pathways by microRNA-24. Mol Ther 2014;22:378-389.

6. Zhao Y, Adjei AA. Targeting angiogenesis in cancer therapy: moving beyond vascular endothelial growth factor. Oncologist 2015;20:660-673.

7. Yamagata K. Soy isoflavones inhibit endothelial cell dysfunction and prevent cardiovascular disease. J Cardiovasc Pharmacol 2019;74:201-209.

8. Chu F, Wang M, Ma H, et al. Simvastatin modulates interaction between vascular smooth muscle cell/macrophage and TNF-alpha-activated endothelial cell. J Cardiovasc Pharmacol 2018;71:268-274.

9. Folkman J. The role of angiogenesis in tumor growth. Semin Cancer Biol 1992;3:65-71.

10. Hanawa K, Ito K, Aizawa K, et al. Low-intensity pulsed ultrasound induces 
angiogenesis and ameliorates left ventricular dysfunction in a porcine model of chronic myocardial ischemia. PLoS One 2014;9:e104863.

11. Liu H, Tao Y, Chen M, et al. 17ß-Estradiol Promotes Angiogenesis of Rat Cardiac Microvascular Endothelial Cells In Vitro. Med Sci Monit 2018;24:2489-2496.

12. Ran $Y$, Ma L, Wang $X$, et al. Design, synthesis, and anti-proliferative evaluation of [1,1'-biphenyl]-4-ols as inhibitor of HUVEC migration and tube formation. Molecules 2012;17:8091-8104.

13. Huang $H$, Zhang $Q$, Liu J, et al. Granulocyte-colony stimulating factor (G-CSF) accelerates wound healing in hemorrhagic shock rats by enhancing angiogenesis and attenuating apoptosis. Med Sci Monit 2017;23:2644-2653.

14. Bartel DP. MicroRNAs: target recognition and regulatory functions. Cell 2009;139:215-233.

15. Li LK, Kong ZH. Screening and functional analysis of micro-RNAs that regulate the expression of the tumor suppression TP53 gene. Eur Rev Med Pharmacol Sci 2018;22:1937-1942.

16. Sayed D, Abdellatif M. MicroRNAs in development and disease. Physiol Rev 2011;91:827-887.

17. Umezu T, Ohyashiki K, Kuroda M, et al. Leukemia cell to endothelial cell communication via exosomal miRNAs. Oncogene 2013;32:2747-2755.

18. Wen Z, Huang W, Feng Y, et al. MicroRNA-377 regulates mesenchymal stem cell-induced antiogenesis in ischemic hearts by targeting VEGF. PLoS One 2014;9:e104666.

19. Chiavacci E, Rizzo M, Pitto L, et al. The zebrafish/tumor xenograft antiogenesis assay as a tool for screening anti-angiogenic miRNAs. 
Cytotechnology 2015;67:969-975.

20. Andersen M, Trapani D, Ravn J, et al. Methylation-associated silencing of micrRNA-126 and its host gene EGFL7 in malignant pleural mesothelioma. Anticancer Res 2015;35:6223-6229.

21. Wei Y, Nazari-Jahantigh M, Neth P, et al. MicroRNA-126, -145 and -155: a therapeutic triad in atherosclerosis? Arterioscler Thromb Vasc Biol 2013;33:449-454.

22. Fitch MJ, Campagnolo L, Kuhnert F, et al. Egfl7, a novel epidermal growth factor, domain gene expressed in endothelial cells. Dev Dyn 2004;230:316-324.

23. Sun $Y$, Bai $Y$, Zhang $F$, et al. miR-126 inhibits non-small cell lung cancer cells proliferation by targeting EGFL7. Biochem Biphys Res Commun 2015;391:1483-1489.

24. Pourgholami MH, Khachigian LM, Fahmy RG, et al. Albendazole inhibits endothelial cell migration, tube formation, vasopermeability, VEGF receptor-2 epxression and suppresses retinal neovascularization in ROP model of angiogenesis. Biochem Biophys Res Commun 2010;397:729-734.

25. Livak KJ, Schmittgen TD. Analysis of relative gene expression data using real-time quantitative PCR and the $2^{-\triangle \Delta \mathrm{ct}}$ method. Methods $2001 ; 25: 402-408$.

26. Li Q, Cheng K, Wang AY, et al. microRNA-126 inhibits tube formation of HUVECs by interacting with EGFL7 and down-regulating PI3K/AKT signaling pathway. Biomed Pharmacother 2019;116:109007.

27. Huang JJ, Shi YQ, Li RL, et al. Angiogenesis effect of therapeutic ultrasound on HUVECs through activation of the PI3K-Akt-eNOS signal pathway. Am J Trans/ Res 2015;7:1106-1115. 
28. Zhang D, Lv FL, Wang GH. Effects of HIF-1 alpha on diabetic retinopathy angiogenesis and VEGF expression. Eur Rev Med Pharmacol Sci 2018;22:5071-4076.

29. Fan C, Yang LY, Wu F, et al. The expression of Egfl7 in human normal tissues and epithelial tumors. Int J Biol Markers 2013;28:71-83.

30. $\mathrm{Hu} \mathrm{MH}$, Ma CY, Wang XM, et al. MicroRNA-126 inhibits tumor proliferation and angiogenesis of hepatocellular carcinoma by down-regulating EGFL7 expression. Oncotarget 2016;7:66922-66934.

31. Yang HH, Chen Y, Gao CY, et al. Protective effects of microRNA-126 on human cardiac microvascular endothelial cells against hypoxia/reoxygenation-induced injury and inflammatory response by activating PI3K/Akt/eNOS signaling pathway. Cell Physiol Biochem 2017;42:506-518.

32. Zhang Q, Zeng S, Quan C, et al. Induction function of miR-126 in survival and proliferation in neural stem cells. Med Sci Monit 2015;21:3023-3027.

33. Sakai E, Miura Y, Suzuki-Kouyama E, et al. A mammalian mirtron miR-1224 promotes tube-formation of human primary endothelial cells by targeting anti-angiogenic factor epsin 2. Sci Rep 2017;7:5541.

34. Andersen M, Grauslund M, Ravn J, et al. Diagnostic potential of miR-126, miR-143, miR-145, and miR-652 in malignant pleural mesothelioma. $J$ Mol Diagn 2014;16:418-430.

35. Hansen TF, Christensen RD, Andersen RF, et al. MicroRNA-126 and epidermal growth factor-like domain 7, an angiogenic couple of importance in metastatic colorectal cancer. Results from the Nordic ACT trial. Br J Cancer 2013;109:1243-1251.

36. Gong C, Fang J, Li G, et al. Effects of microRNA-126 on cell proliferation, 
apoptosis and tumor angiogenesis via the down-regulating ERK signaling pathway by targeting EGFL7 in hepatocellular carcinoma. Oncotarget $2017 ; 8: 52527-52542$.

37. Cheng HW, Chen YF, Wong JM, et al. Cancer cells increase endothelial cell tube formation and survival by activating the PI3K/Akt signalling pathway. $J$ Exp Clin Cancer Res 2017;36:27.

38. Casciaro M, Di Salvo E, Brizzi $T$, et al. Involvement of miR-126 in autoimmune disorders. Clin Mol Allergy 2018;16:11.

39. Pishavar E, Behravan J. miR-126 as a therapeutic agent for diabetes mellitus. Curr Pharm Des 2017;23:3309-3314.

40. He X, Xu J. miR-126: a potential biomarker for coronary artery disease? Cardiology 2019;142:2-3.

41. Tuaeva NO, Falzone L, Porozov YB, et al. Translational application of circulating DNA in Oncology: review of the last decades achievements. Cells 2019;8:1251.

42. Sun LL, Li WD, Lei FR, et al. The regulatory role of microRNAs in angiogenesis-related diseases. J Cell Mol Med 2018;22:4568-4587.

43. Landskroner-Eiger S, Moneke I, Sessa WC. miRNA as modulators of angiogenesis. Cold Spring Harb Perspect Med 2013;3:a006643. 
Table I . Gene sequences for the RT-PCR.

\begin{tabular}{|c|c|c|c|}
\hline Gene & & Sequences & Length (bp) \\
\hline \multirow{2}{*}{$\operatorname{miR}-126$} & Forward & 5'-TGGTCGTACCGTGAGTA-3' & \multirow{2}{*}{146} \\
\hline & Reverse & 5'-GCGCGT GAGCAGGCTGGAGAA -3' & \\
\hline \multirow{2}{*}{ EGFL7 } & Forward & 5'-GGGATGGCAGGGAGATACTTG-3' & \multirow{2}{*}{194} \\
\hline & Reverse & 5'-CTGGCGTGGGACTTGGTG-3' & \\
\hline \multirow{2}{*}{$\beta$-actin } & Forward & 5'-CCCATCTATGAGGGTTACGC-3' & \multirow{2}{*}{150} \\
\hline & Reverse & 5'-TTTAATGTCACGCACGATTTC-3' & \\
\hline
\end{tabular}


A

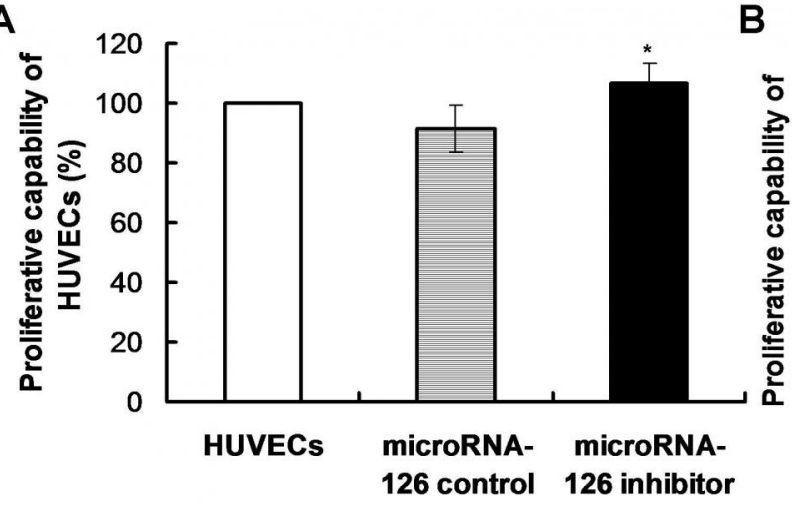

C

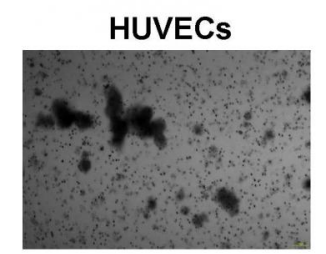

microRNA-126 inhibitor

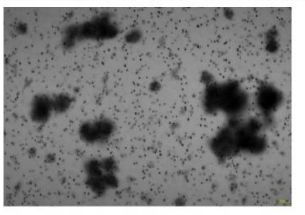

B

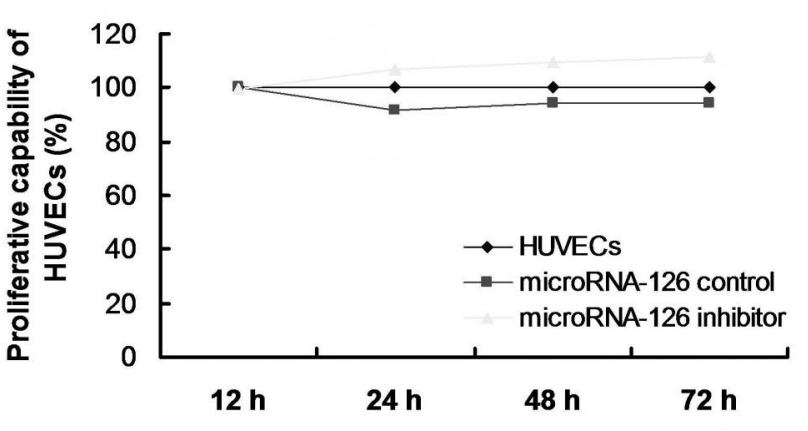

D

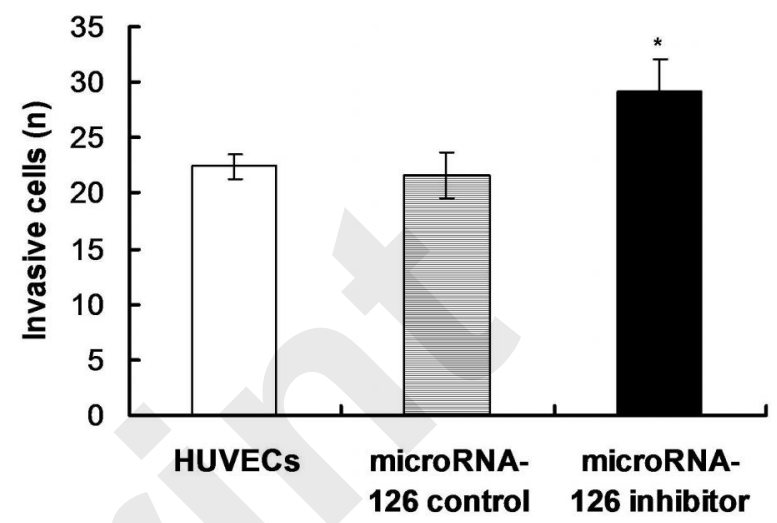

Figure 1. Effects of microRNA-126 inhibitor administration on proliferative capability and invasive ability of HUVECs. A. Statistical analysis for microRNA-126 inhibitor's effects on proliferative capability. B. Proliferative effect of microRNA-126 on HUVECs with a timedependent manner. C. Images of the invasive HUVECs. D. Statistical analysis for microRNA-126 induced cell invasion. ${ }^{*} p<0.05$ vs. microRNA-126 control group.

Magnification, 100x. 
A

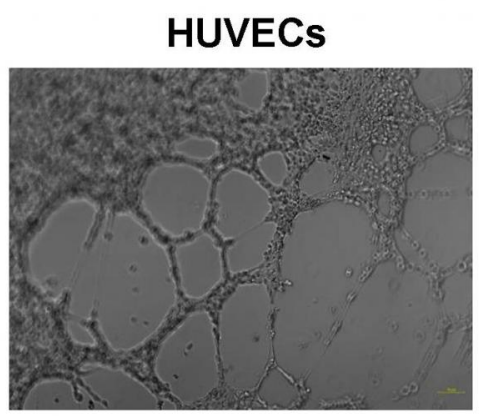

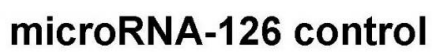

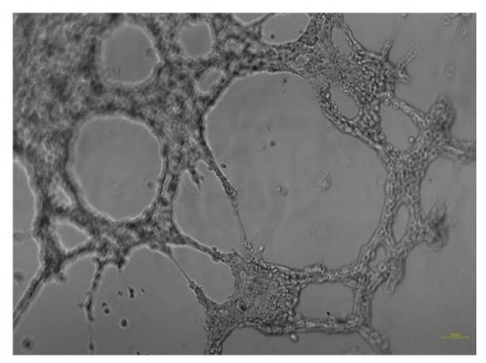

microRNA-126 inhibitor

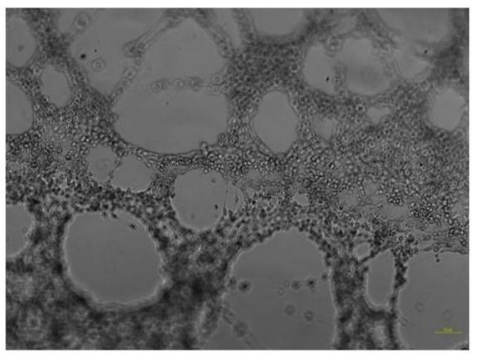

B

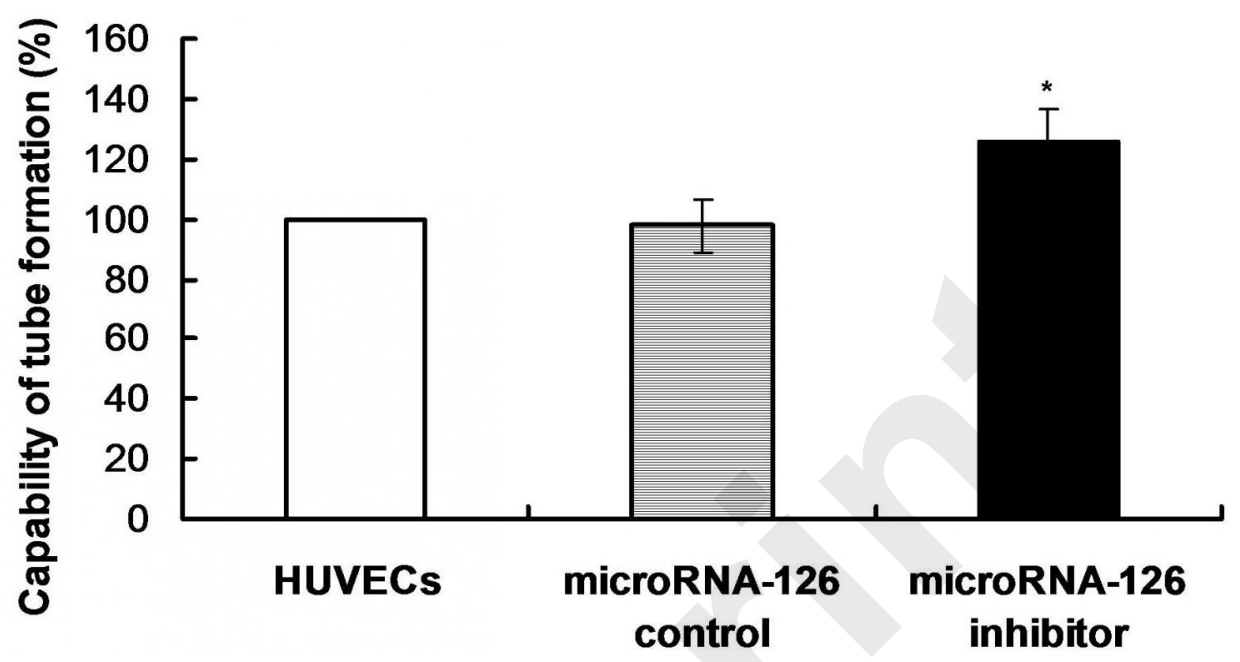

Figure 2. microRNA-126 inhibitor transfection induced the formation of tube. A. Tube formation images in the cultured HUVECs. B. Statistical analysis of tube formation. ${ }^{*} p<0.05$ vs. microRNA-126 control group. 
A

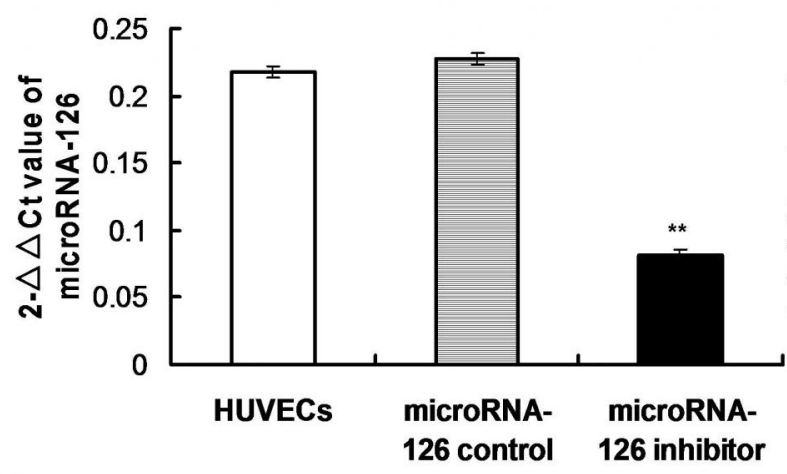

B

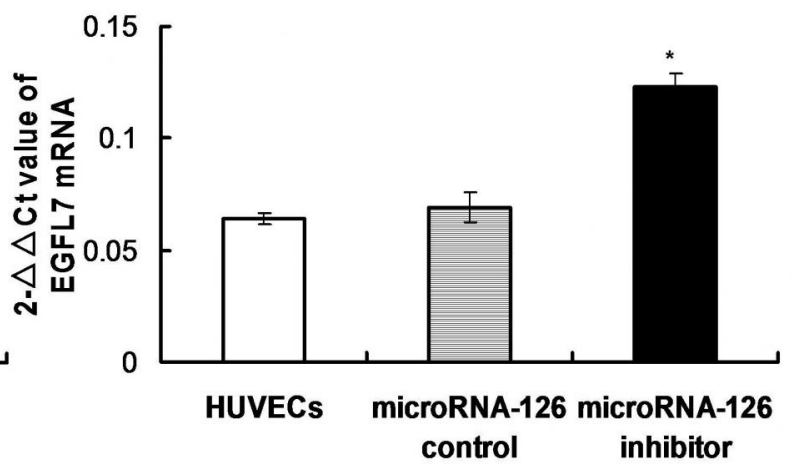

Figure 3. microRNA-126 inhibitor administration enhanced the EGFL7 mRNA expression. A. Statistical analysis of microRNA-126 mRNA expression. B. Statistical analysis of EGFL7 mRNA expression. ${ }^{*} p<0.05$ vs. microRNA-126 control group. 
A
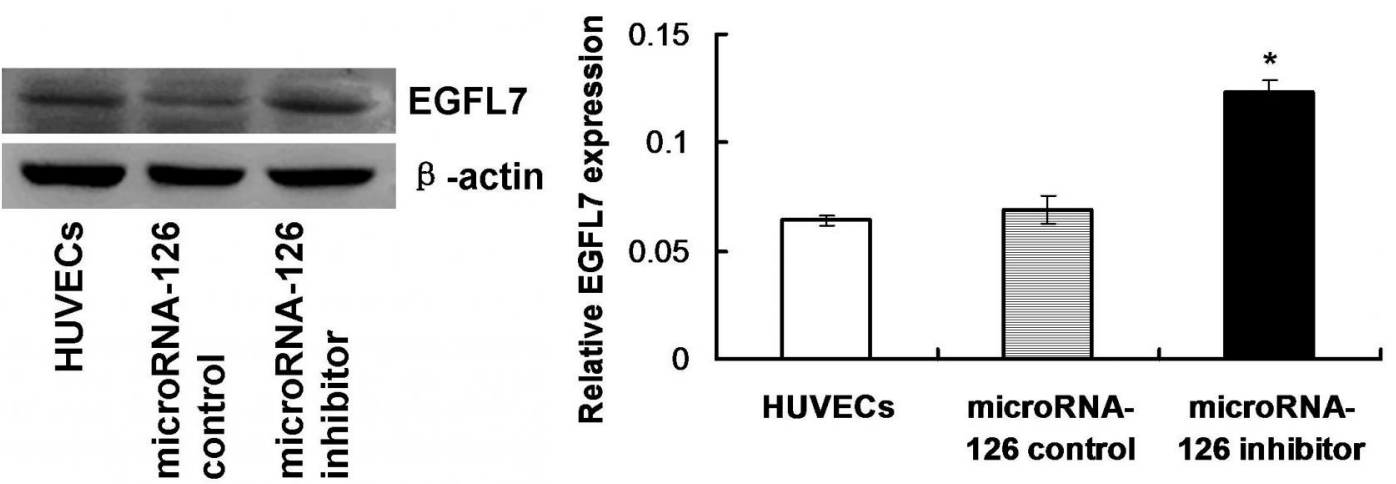

B

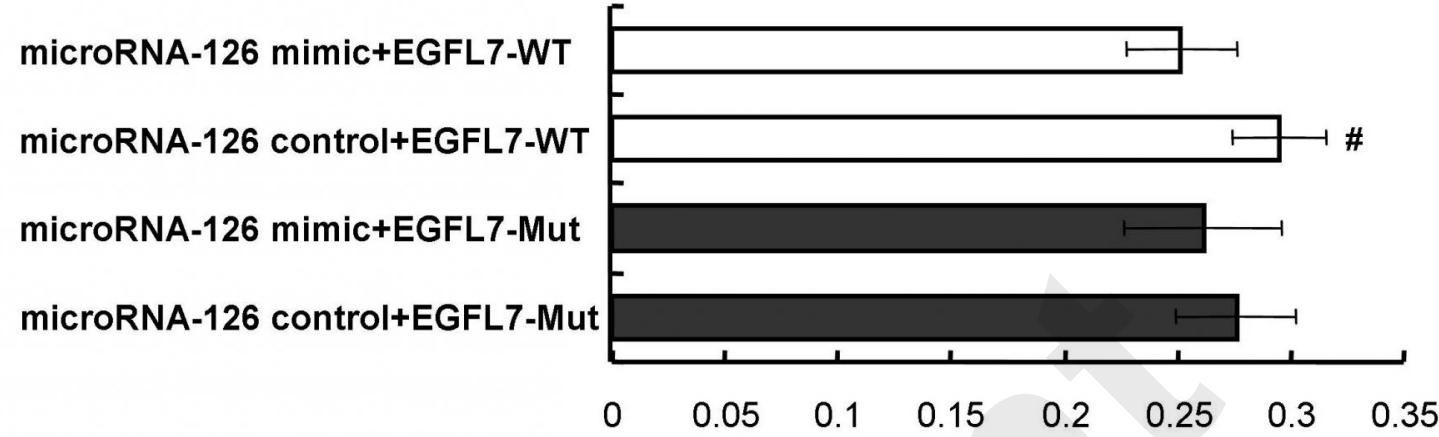

C

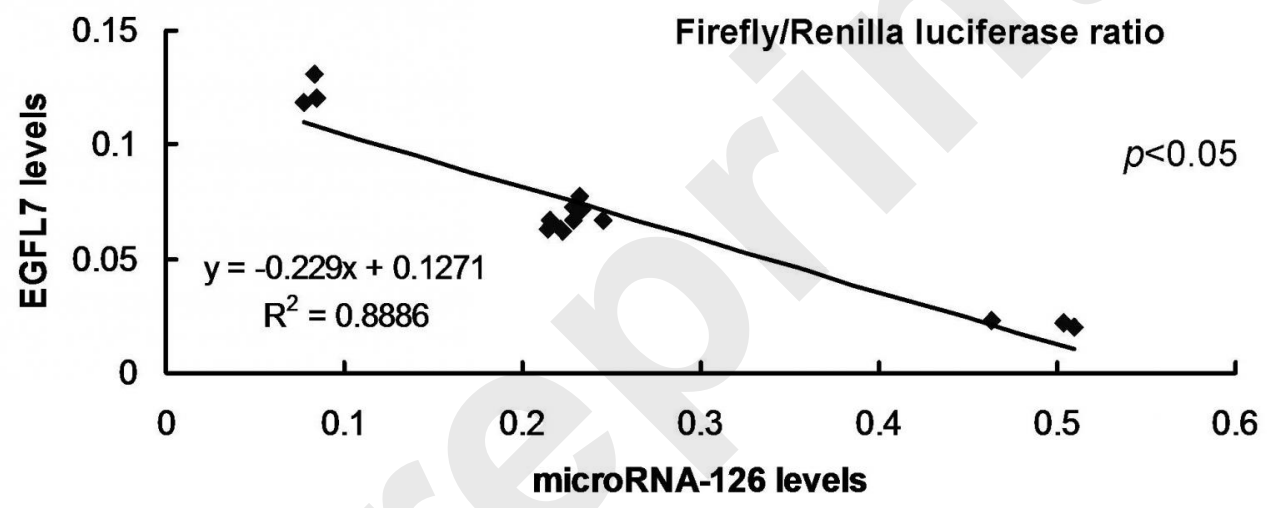

Figure 4. Relationship between microRNA-126 and EGFL7 molecule. A. Determination for effects of microRNA-126 inhibitor administration on EGFL7 expression using western blotting assay. B. Dual-luciferase assay for evaluating interaction between microRNA-126 gene and EGFL7 gene. C. Correlation between microRNA-126 and EGFL7 using Linear Regression analysis. ${ }^{*} p<0.05$ vs. microRNA-126 control group. $\# p<0.05$ vs. microRNA-126 mimic+EGFL7-WT group. 
A
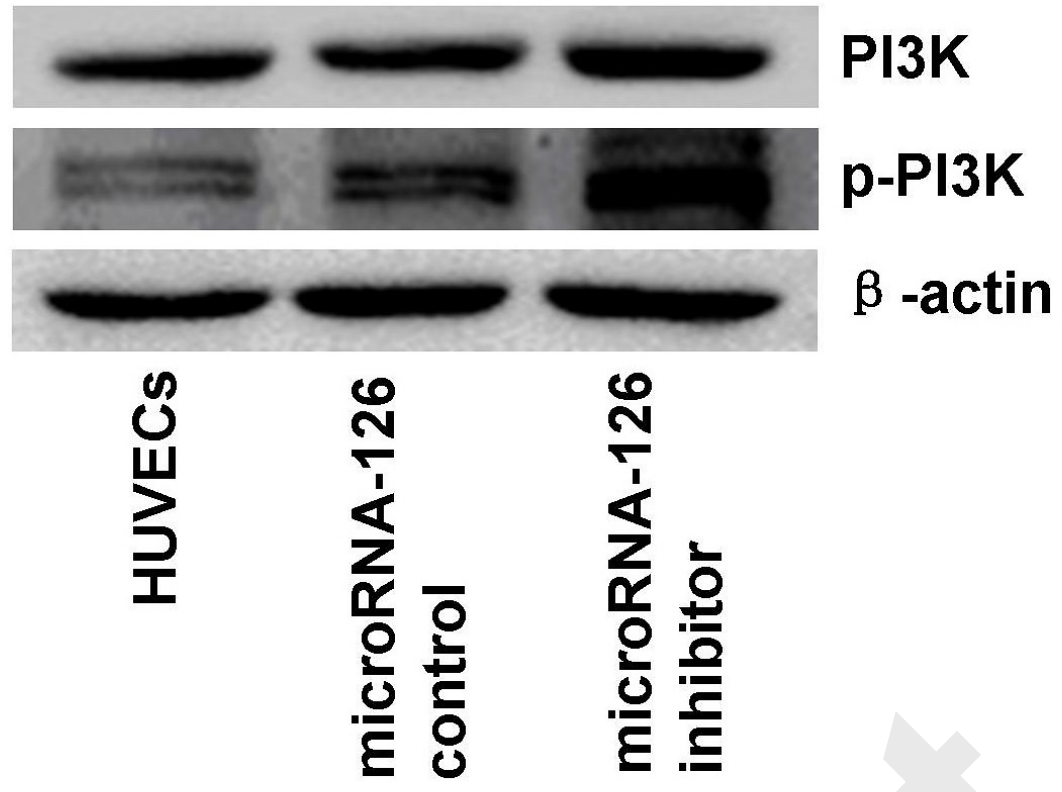

B

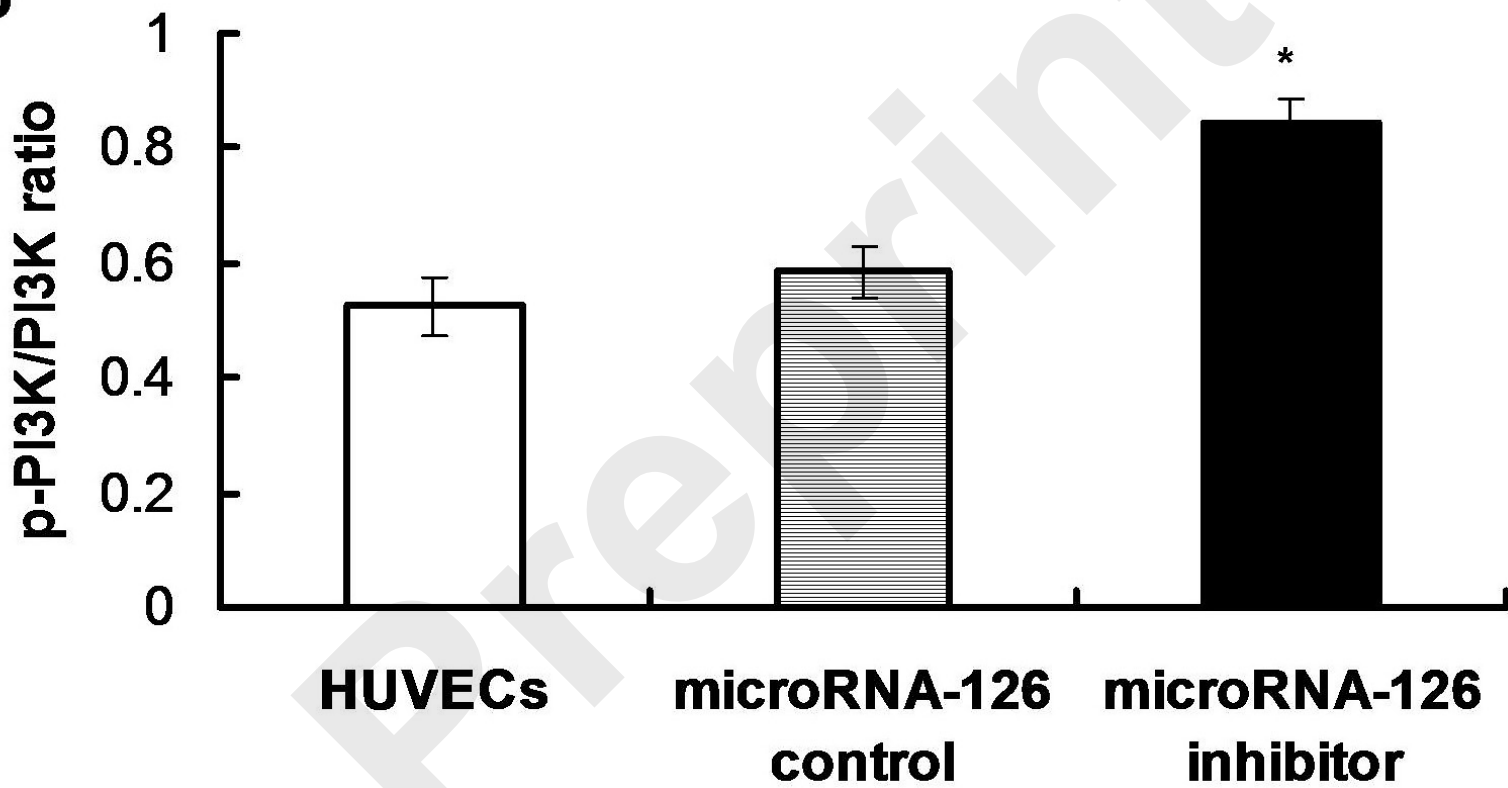

Figure 5. Evaluation for enhancive effects of microRNA-126 inhibitor administration on $p$ $\mathrm{PI} 3 \mathrm{~K}$ and PI3K expression using western blotting assay. A. Western blotting images for $p-$ $\mathrm{PI} 3 \mathrm{~K}$ and PI3K expression. B. Statistical analysis for ratio of $\mathrm{p}-\mathrm{PI} 3 \mathrm{~K} / \mathrm{PI} 3 \mathrm{~K}$. ${ }^{*} \mathrm{p}<0.05$ vs.

microRNA-126 control group. 


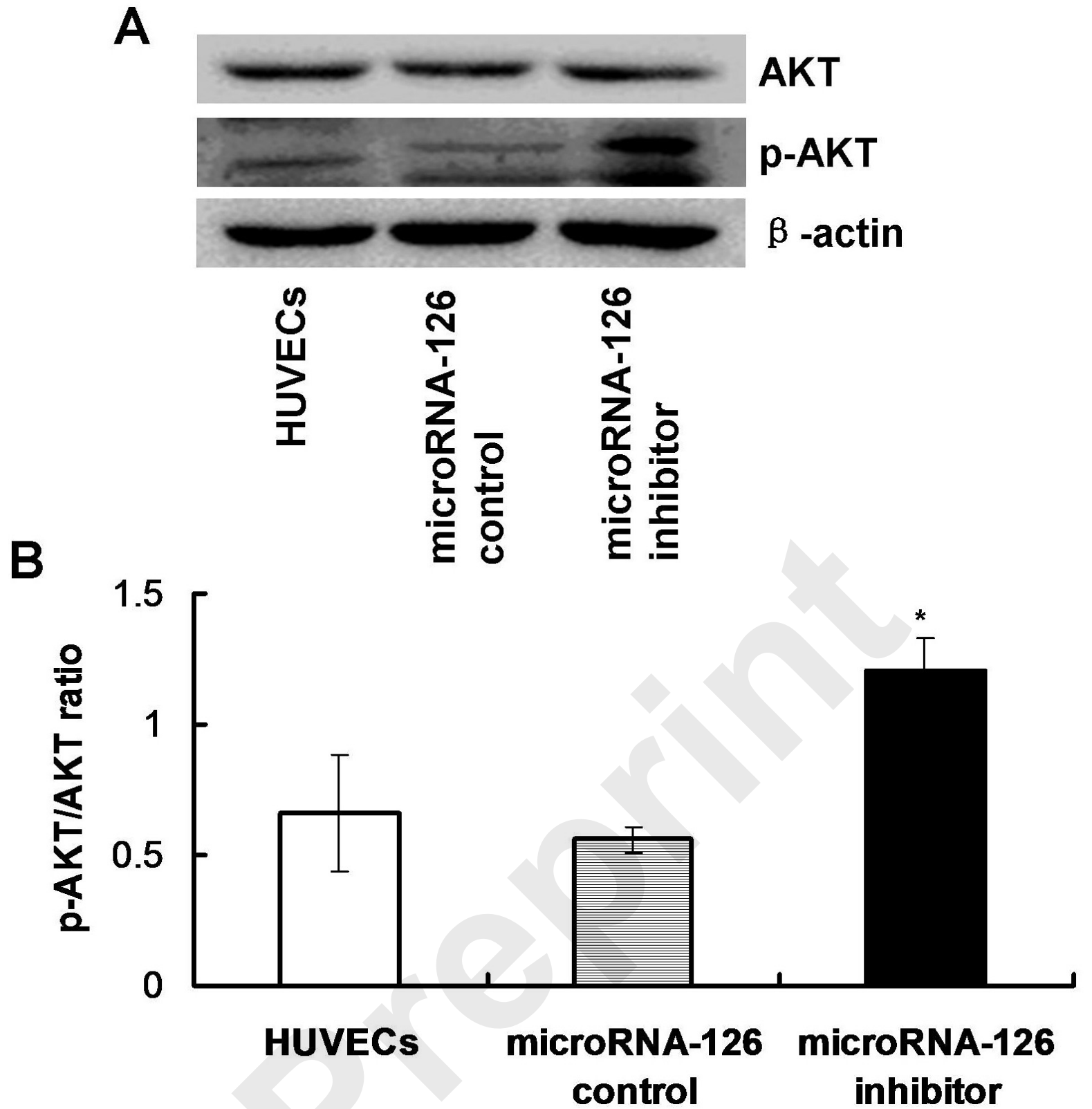

Figure 6. microRNA-126 silence promoted p-AKT and AKT expression using western blotting assay. A. Western blotting images for $p-A K T$ and AKT expression. B. Statistical analysis for ratio of $p-A K T / A K T .{ }^{*} p<0.05$ vs. microRNA-126 control group. 